\title{
Neutrophil apoptosis mediated by nicotinic acid receptors (GPR109A)
}

\author{
G Kostylina ${ }^{1}$, D Simon ${ }^{2}$, MF Fey ${ }^{3}$, S Yousefi ${ }^{1}$ and HU Simon ${ }^{\star, 1}$
}

G protein-coupled receptor (GPR)109A (HM74A) is a $G_{i}$ protein-coupled receptor, which is activated by nicotinic acid (NA), a lipidlowering drug. Here, we demonstrate that mature human neutrophils, but not eosinophils, express functional GPR109A receptors. The induction of the GPR109A gene appears to occur late in the terminal differentiation process of neutrophils, since a mixed population of immature bone marrow neutrophils did not demonstrate evidence for its expression. NA accelerated apoptosis in cultured neutrophils in a concentration-dependent manner, as assessed by phosphatidylserine redistribution, caspase-3 activation, and DNA fragmentation assays. The pro-apoptotic effect of NA was abolished by pertussis toxin, which was used to block $G_{i}$ proteins, suggesting a receptor-mediated mechanism. Activation of GPR109A by NA resulted in decreased levels of cyclic adenosine monophosphate (cAMP), most likely due to $G_{i}$-mediated inhibition of adenylyl cyclase activity. NAinduced apoptosis was reversed by the addition of cell-permeable cAMP, pointing to the possibility that reduced cAMP levels promote apoptosis in neutrophils. Distal mechanism involved in this process may include the post-translational modification of members of the Bcl-2 family, such as dephosphorylation of pro-apoptotic Bad and antiapoptotic Mcl-1 proteins. Taken together, following maturation in the bone marrow, neutrophils express functional GPR109A receptors, which might be involved in the regulation of neutrophil numbers. Moreover, this study identified a new cellular target of NA and future drugs activating GPR109A receptors, the mature neutrophil.

Cell Death and Differentiation (2008) 15, 134-142; doi:10.1038/sj.cdd.4402238; published online 12 October 2007

The $\mathrm{G}_{i}$ protein-coupled receptor GPR109A (HM74A in humans; PUMA-G in mice) has been identified as a receptor for nicotinic acid (NA). ${ }^{1,2}$ NA is used to modify lipoprotein profiles in humans, resulting in a beneficial modification of multiple cardiovascular risk factors. ${ }^{3}$ This highly desirable therapeutic profile of NA is due to its direct activation of GPR109A on the surface of adipocytes. ${ }^{1}$ However, GPR109A mRNA is also highly expressed in spleen and lung tissues, ${ }^{2}$ but it remains unclear which cells indeed express the receptor and whether it is functional. Recently, it has been shown that interferon- $\gamma$ is able to induce GPR109A receptors in macrophages, and that NA is subsequently able to stimulate prostaglandin E2 and D2 synthesis in these cells. ${ }^{4}$ The identification of functional GPR109A receptors in a cell outside adipose tissue raised the question whether additional cell types carry GPR109A receptors, which might also be targeted during therapy with NA.

Neutrophils are a critical component of the innate immune system with several effector and immunoregulatory functions. Their numbers are largely regulated by production in the bone marrow and death, which is commonly apoptosis. ${ }^{5}$ Delayed neutrophil apoptosis has been reported in association with several infectious diseases and is considered as a major mechanism of tissue neutrophilia. Overexpression of neutro- phil survival factors, such as granulocyte colony-stimulating factor (G-CSF) and granulocyte-macrophage colonystimulating factor (GM-CSF), play a major role in this process. ${ }^{6-9}$ These survival cytokines have been described to block the mitochondria-dependent death pathway in neutrophils. ${ }^{8}$ This is also reflected by the observation that these cytokines regulate the expression of $\mathrm{Bcl}-2$ family members in these cells. ${ }^{5}$

In chronic inflammatory diseases, persistent accumulation of inflammatory cells may lead to increased release of toxic intracellular products, leading to tissue injury and subsequent remodeling with potential consequences on organ function. Therefore, it is important to study how inflammation normally resolves and under which conditions this process does not work. Although some surface receptors on neutrophils have been identified that transduce death signals, such as Fas ${ }^{10}$ and Siglec- $9,{ }^{11}$ other death ligands may additionally contribute to the limitation of neutrophil numbers under inflammatory conditions. Additional knowledge in this field may provide novel approaches to anti-inflammatory pharmacotherapy.

In this article, we report the expression of functional GPR109A receptors on the surface of neutrophils, supporting the concept that they play a role in immunity and inflammation.

\footnotetext{
${ }^{1}$ Department of Pharmacology, University of Bern, Bern, Switzerland; ${ }^{2}$ Department of Dermatology, University of Bern, Bern, Switzerland and ${ }^{3}$ Department of Medical Oncology, University of Bern, Bern, Switzerland

${ }^{*}$ Corresponding author: HU Simon, Department of Pharmacology, University of Bern, Friedbühlstrasse 49, Bern CH-3010, Switzerland. Tel: +41316323281 ;

Fax: + 4131632 4992; E-mail: hus@pki.unibe.ch

Keywords: apoptosis; Bad; cAMP; neutrophils; nicotinic acid

Abbreviations: $\left[\mathrm{Ca}^{2+}\right]$, cytosolic-free calcium concentrations; cAMP, cyclic adenosine monophosphate; GAPDH, glyceraldehyde-3-phosphate dehydrogenase; G-CSF, granulocyte colony-stimulating factor; GM-CSF, granulocyte-macrophage colony-stimulating factor; GPR, G protein-coupled receptor; mAb, monoclonal antibody; NA, nicotinic acid; PKA, protein kinase A; PS, phosphatidylserine; PTX, pertussis toxin

Received 26.4.07; revised 20.8.07; accepted 10.9.07; Edited by P Vandenabeele; published online 12.10.07
} 
Moreover, GPR109A receptors are linked to a pro-apoptotic pathway in these cells, suggesting that NA may also mediate anti-inflammatory effects besides its inhibitory function on adipocyte lipolysis.

\section{Results}

Mature neutrophils, but not eosinophils or immature neutrophils, express GPR109A surface receptors. We investigated GPR109A surface expression on purified granulocyte population using indirect immunofluorescence and flow cytometric analysis. Freshly purified mature blood neutrophils, but not eosinophils or immature bone marrow neutrophils, expressed detectable GPR109A surface expression (Figure 1a). In addition, immature neutrophilic HL-60 cells were also GPR109A negative. Stimulation of mature neutrophils with GM-CSF slightly increased GPR109A expression (data not shown). Expression of GPR109A on mature neutrophils was confirmed using confocal microscopy. Both indirect immunofluorescence analysis and direct staining with FITC-conjugated NA demonstrated ring-like staining patterns, suggesting that GPR109A is predominantly expressed on the cell surface of these cells (Figure 1b).

Functional GPR109A receptors are present on mature neutrophils, but not on eosinophils or immature neutrophils. Expression of a functional GPR109A receptor

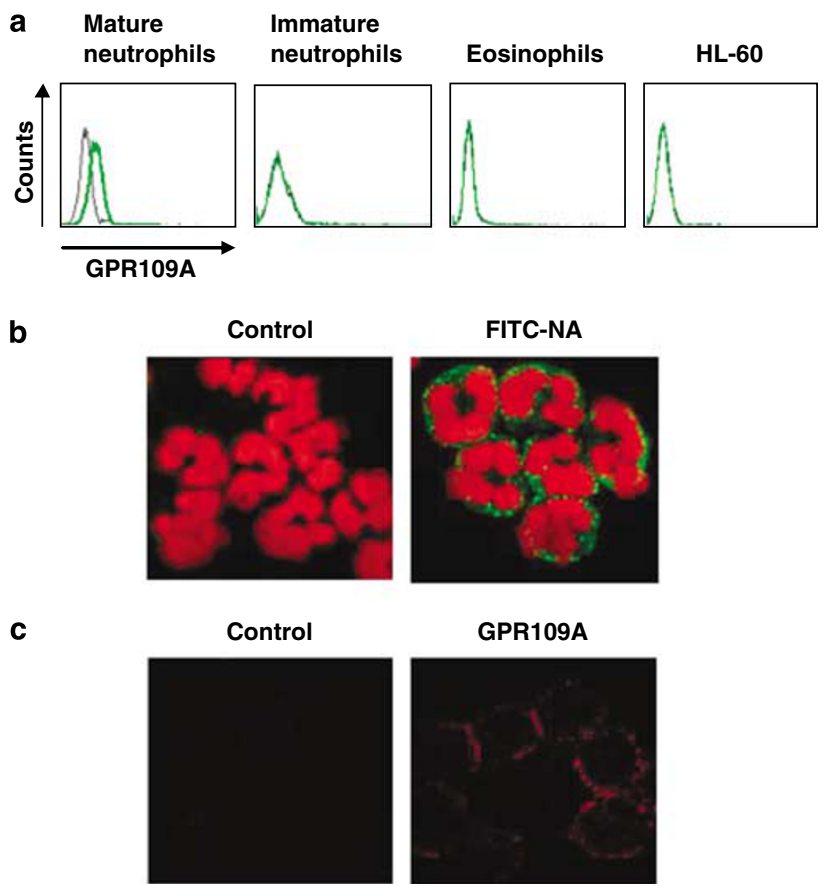

Figure 1 Mature blood neutrophils, but not immature bone marrow neutrophils or mature blood eosinophils, express the NA receptor GPR109A on their surface. (a) Flow cytometry. Cells were stained with control (black) or anti-GPR109A (green) mAbs. (b and c) Confocal microscopy. Neutrophils were stained with FITCconjugated NA and anti-GPR109A mAb, respectively. Nuclei were stained with PE in (b). Results in each panel are representative of at least four independent experiments was assessed by the ability of NA to specifically induce increases in cytosolic-free calcium concentrations $\left(\left[\mathrm{Ca}^{2+}\right]_{\mathrm{i}}\right){ }^{1}$ Addition of NA to fura-2-loaded mature neutrophils led to rapid and transient changes in $\left[\mathrm{Ca}^{2+}\right]_{i}$ (Figure 2a). Peak calcium levels were observed within 1 min of addition of NA. Levels rapidly declined thereafter reaching baseline levels within $5 \mathrm{~min}$. The increases in $\left[\mathrm{Ca}^{2+}\right]_{i}$ were concentration dependent in the range of $10^{-7}-10^{-3} \mathrm{M}$ (Figure 2a). Furthermore, triggering of changes in $\left[\mathrm{Ca}^{2+}\right]_{i}$ were caused by NA binding to its specific receptor because nicotinamide, a structurally related compound, which does not bind to GPR109A receptors, ${ }^{12}$ had no effect on $\left[\mathrm{Ca}^{2+}\right]_{i}$ at concentrations ranging from $10^{-7}$ to $10^{-3} \mathrm{M}$ (data not shown). Moreover, immature bone marrow neutrophils, mature blood eosinophils, and HL-60 cells, which all did not express detectable levels of GPR109A surface protein, did not respond with increases in $\left[\mathrm{Ca}^{2+}\right]_{i}$ following addition of NA. In contrast, addition of UTP, a ligand of $\mathrm{P}_{2} \mathrm{Y}_{2}$ receptors expressed in hematopoietic cells including CD34 + stem cells, ${ }^{13}$ led to rapid and transient changes in $\left[\mathrm{Ca}^{2+}\right]_{i}$ (Figure 2b), demonstrating that the cells were properly loaded and responsive, in principle.

To complement our studies of purified cell populations, we utilized a second functional approach analyzing $\left[\mathrm{Ca}^{2+}\right]_{i}$ changes in single mature neutrophils. Interestingly, all cells responded similarly following activation with NA, regarding time and magnitude of the change in $\left[\mathrm{Ca}^{2+}\right]_{\mathrm{i}}$ (Figure $2 \mathrm{c}$ ). These data suggested that all mature neutrophils express functional GPR109A receptors and also excluded the possibility that the NA-mediated effects on $\left[\mathrm{Ca}^{2+}\right]_{i}$ seen using the bulk spectrofluorometric assay were due to GPR109A-expressing contaminating cells.

Pertussis toxin (PTX) is a known inhibitor of $G_{i}$ proteins. Pre-incubation of mature neutrophils with PTX inhibited NAinduced increases in $\left[\mathrm{Ca}^{2+}\right]_{i}$ in a concentration- and timedependent manner (Figure 2d). In contrast, UTP-induced increases in $\left[\mathrm{Ca}^{2+}\right]_{i}$ were not affected by PTX, consisting with the view that $P 2 Y_{2}$ receptors are able to stimulate, besides $G_{i}$, additional $\mathrm{G}$ proteins. ${ }^{14}$ Taken together, these data suggest that mature neutrophils express functional GPR109A receptors, which can be blocked by PTX. Moreover, increases in $\left[\mathrm{Ca}^{2+}\right]_{\mathrm{i}}$ induced by $10^{-5} \mathrm{M} \mathrm{NA}$ are an accurate and sensitive indicator for the presence of functional GPR109A receptors.

NA accelerates apoptosis in cultured mature neutrophils, but not in eosinophils or immature neutrophils. Mature neutrophils and eosinophils are known short-lived cells and undergo rapid apoptosis in vitro. $^{5}$ In contrast, immature neutrophils exhibit a much longer life-span in vitro, although they also undergo cell death at later time points. ${ }^{15,16}$ Addition of NA to purified cell populations resulted in a detectable cytotoxic effect in mature neutrophils, but not in eosinophils or immature neutrophils (including HL-60 cells), in a concentration-dependent manner in 24-h cultures (Figure 3a). Longer culture times (48-h) revealed same results, except that spontaneous death of mature granulocytes was more advanced (data not shown). Concentrations of $10^{-5}$ and $10^{-4} \mathrm{M}$ NA significantly accelerated the known spontaneous death in mature neutrophils. 
a

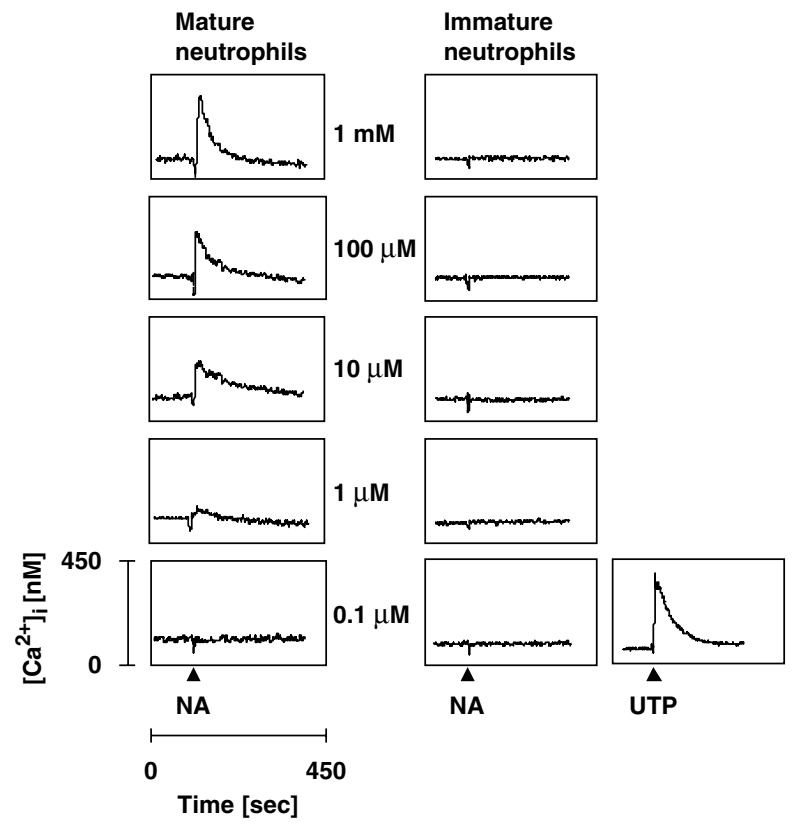

b

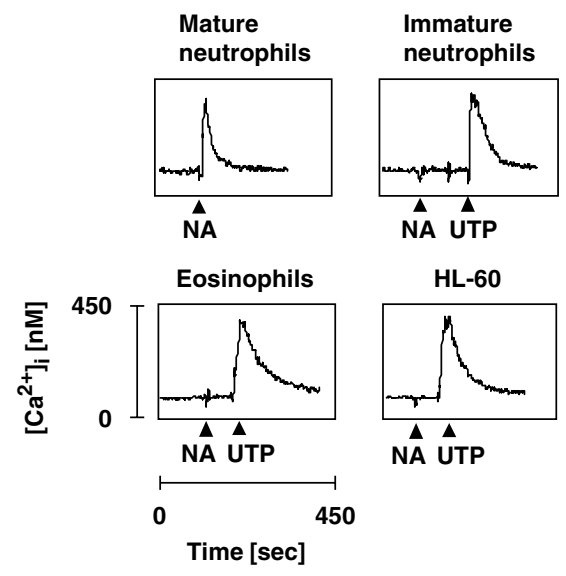

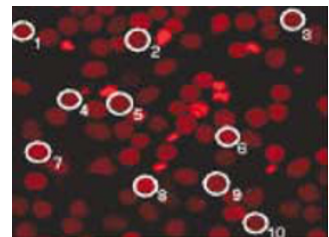
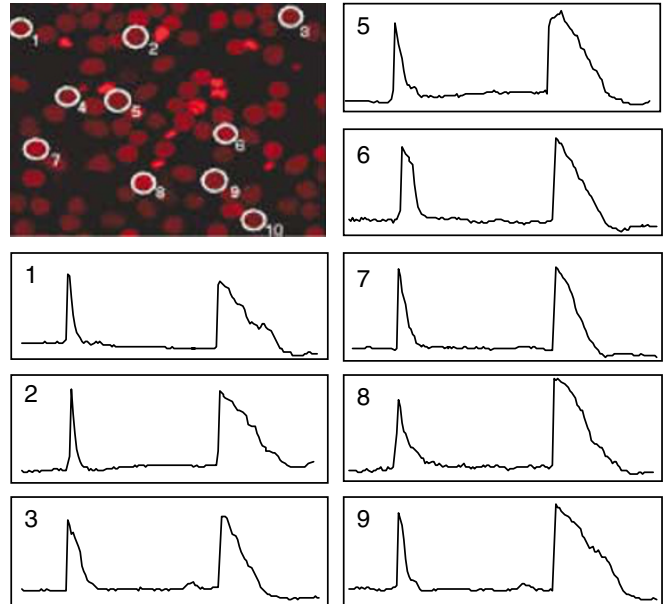

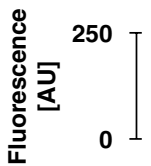
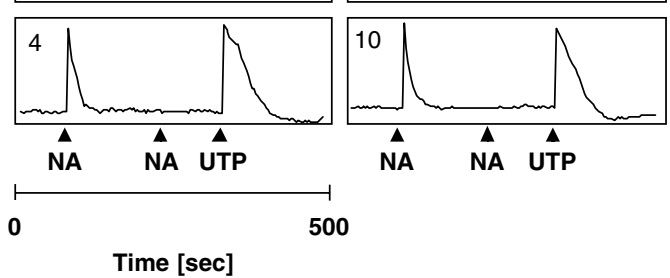

Time $[\mathbf{s e c}]$

d PTX $[\mathrm{ng} / \mathrm{ml}]$
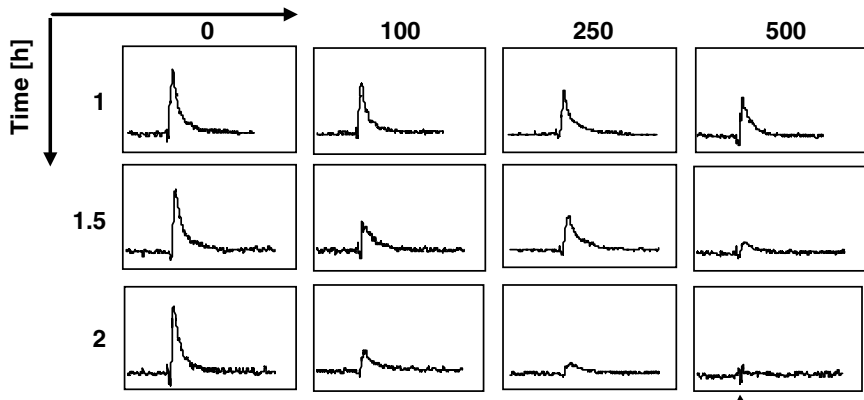

3
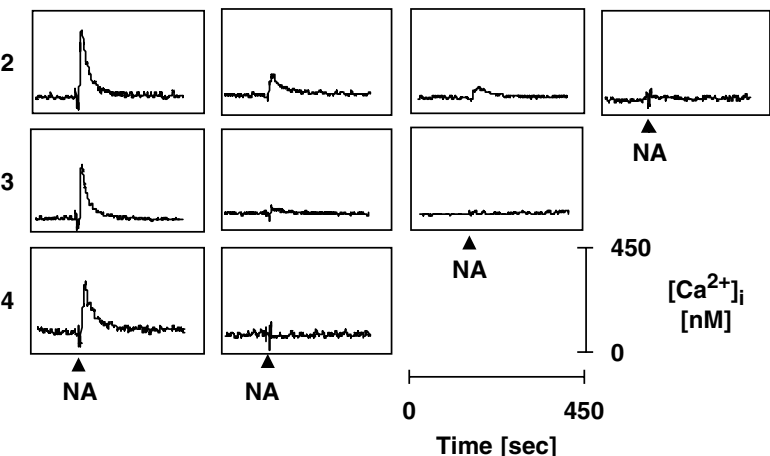

Figure 2 Mature blood neutrophils, but not immature bone marrow neutrophils or mature blood eosinophils, express functional GPR109A. (a) Concentration-dependent increases in $\left[\mathrm{Ca}^{2+}\right]_{i}$ in mature neutrophils (left). NA had no effect on $\left[\mathrm{Ca}^{2+}\right]_{i}$ in immature neutrophils (right). Positive control experiments were performed using UTP. (b) Functional GPR109A receptors were detectable in mature blood neutrophils, but not in their precursors in the bone marrow, HL-60 cells or in mature blood eosinophils. Positive control experiments were performed using UTP. NA was added at $100 \mu \mathrm{M}$. (c) Single-cell analysis. Changes in $\left[\mathrm{Ca}^{2+}\right]_{i}$ were analyzed in 10 single blood neutrophils. A second NA stimulation did not result in increases in $\left[\mathrm{Ca}^{2+}\right]_{\text {, }}$, suggesting homologous desensitization. (d) PTX inhibited increases in $\left[\mathrm{Ca}^{2+}\right]_{\mathrm{i}}$ in a concentration- and time-dependent manner. Positive control experiments were performed using UTP (data not shown). Representative examples of at least four independent experiments are shown in each panel. NA and UTP $(20 \mu \mathrm{M})$ were added where indicated $(\mathbf{\Lambda})$

The death efficacy of NA was similar to that obtained with optimal concentrations of anti-Fas monoclonal antibody $(\mathrm{mAb})$, a known trigger of neutrophil cell death. ${ }^{10}$ Moreover, since cytokine-mediated delayed apoptosis of mature neutrophils plays an important role under inflammatory conditions, ${ }^{5}$ we were interested in the effect of NA in the presence of GM-CSF. Interestingly, NA completely abolished the cytoprotective effect of GM-CSF in vitro (Figure 3b).

The type of death, which was induced by NA was apoptosis, as assessed by phosphatidylserine (PS) redistribution (Figure 3c, upper panel), DNA fragmentation (Figure 3d), and morphological analysis (data not shown). The proapoptotic effect of NA was blocked by pre-incubation of 
a

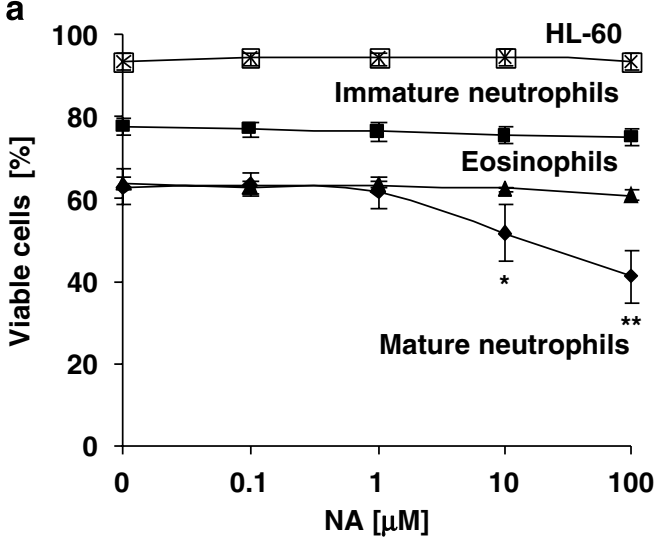

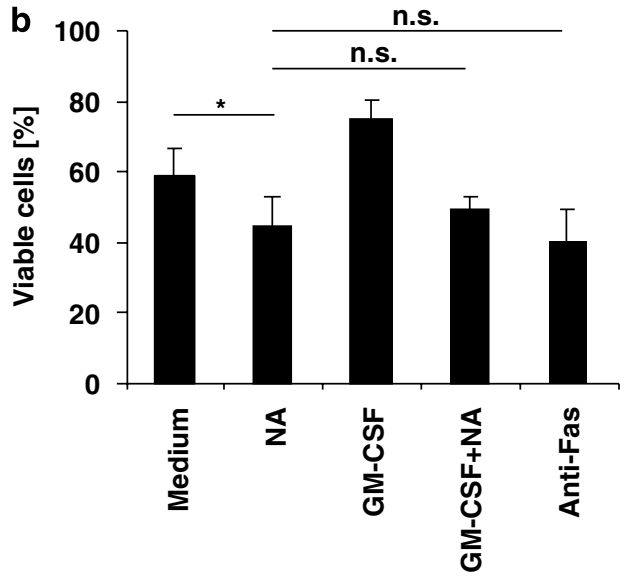

c
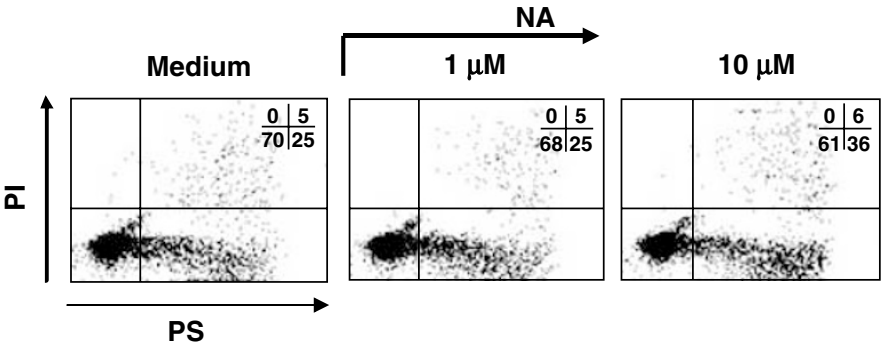

$100 \mu \mathrm{M}$
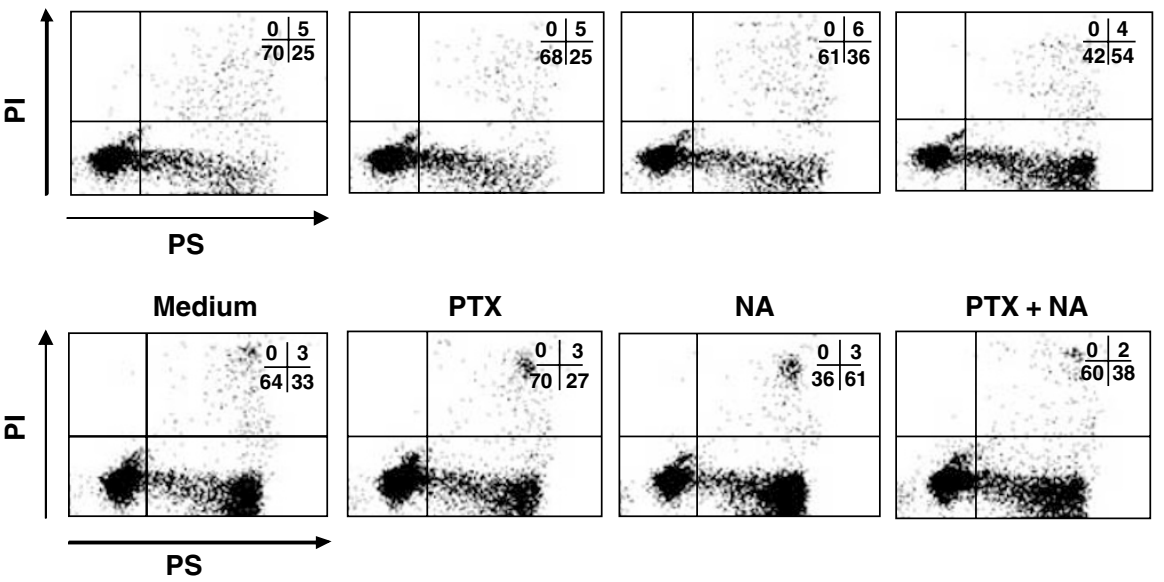

d

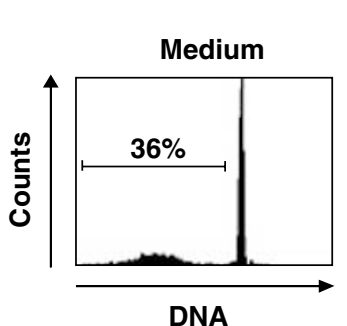

NA
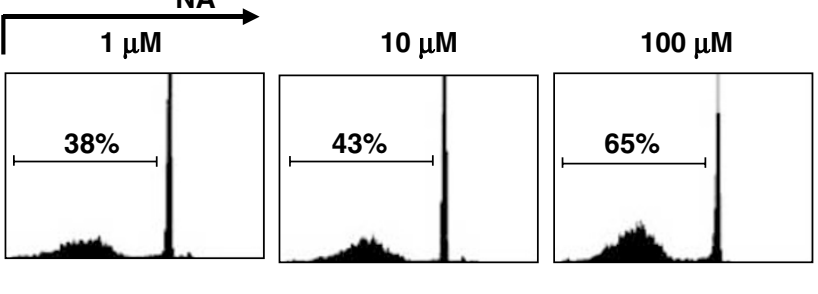

Figure 3 NA accelerates spontaneous neutrophil apoptosis. (a) NA induced death in mature neutrophils in a concentration-dependent manner. In contrast, NA had no cytotoxic effect on blood eosinophils, immature neutrophils, or $\mathrm{HL}-60$ cells. Results of 24-h cultures are shown. Data are expressed as means \pm S.D. of 4-10 independent experiments. ${ }^{*} P<0.05$; ${ }^{*} P<0.01$. Similar data were observed in 48-h cultures. (b) The death efficacy of $100 \mu \mathrm{M} \mathrm{NA}$ and $1 \mu \mathrm{g} / \mathrm{ml}$ anti-Fas mAb on blood neutrophils was similar. Moreover, NA completely abolished the anti-death effect of $25 \mathrm{ng} / \mathrm{ml} \mathrm{GM-CSF}$. Results of 24-h cultures are shown $(n=3)$. Data are expressed as means \pm S.D. ${ }^{*} P<0.05$. (c) NA stimulation induced PS redistribution in blood neutrophils indicative of the induction of apoptosis in a concentration-dependent manner (upper panel). PTX $(500 \mathrm{ng} / \mathrm{ml}$ for $2 \mathrm{~h})$ abolished this effect. Quantitative analysis is shown in percent at the top right corner of each dot blot. The panels show one representative experiment out of three (8-h cultures). (d) NA induced DNA fragmentation in a concentration-dependent manner. The relative number of apoptotic blood neutrophils is indicated by the bracket in each subpanel. One representative experiment out of three is shown (14-h cultures)

neutrophils with PTX, suggesting that it is GPR109A-receptormediated (Figure 3c, lower panel). Moreover, caspase-3, a critical effector caspase in neutrophil apoptosis, ${ }^{17}$ is cleaved $4 \mathrm{~h}$ upon addition of NA into the apparent active enzyme (17-kDa fragment), whereas non-treated neutrophils do not demonstrate any detectable cleavage of caspase-3 at this time point (Figure 4a). We also measured increased enzy- matic activity of caspase-3 in 12-h neutrophil cultures in the presence of NA compared to non-treated cells at this time point or freshly isolated neutrophils (Figure 4b).

The involvement of caspases was further supported by the observation that the pan-caspase inhibitor Z-VAD-fmk as well as the caspase-3 inhibitor z-DEVD-fmk blocked both spontaneous and NA-accelerated neutrophil death (Figure 4c). The 


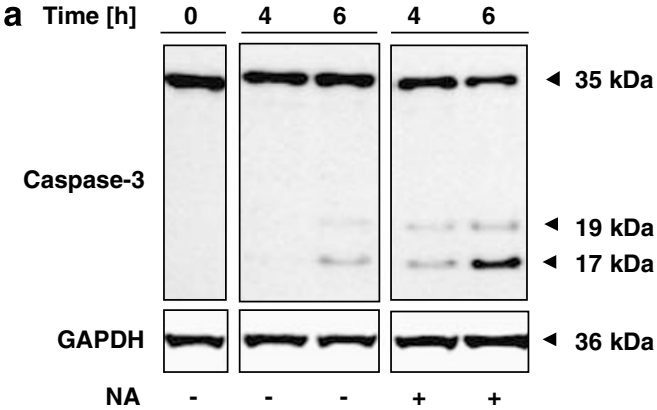

b

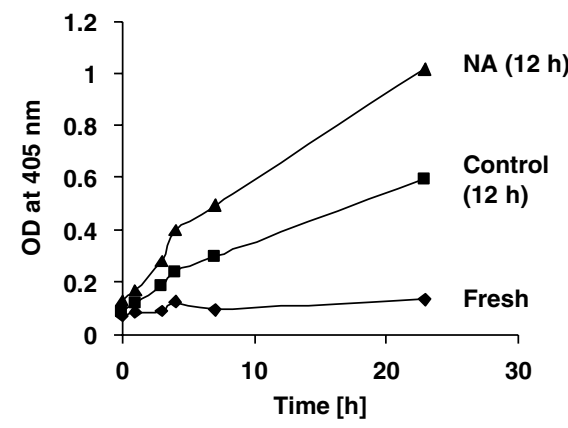

C

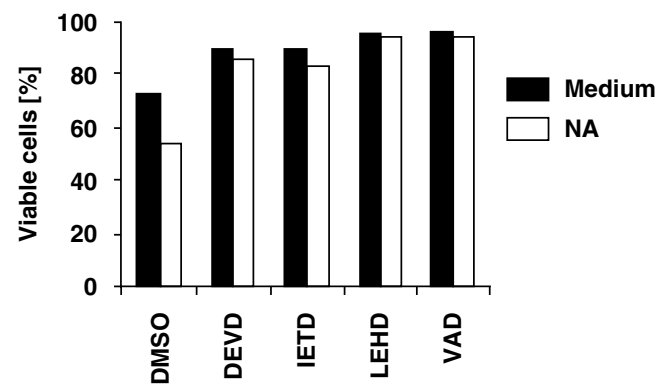

Figure 4 NA-mediated neutrophil apoptosis is caspase-dependent. (a) The induction of neutrophil apoptosis by NA was associated with the rapid occurrence of the $17-\mathrm{kDa}$ caspase-3 cleavage product (detectable in 2-h cultures). (b) Spontaneous neutrophil apoptosis was associated with the induction of caspase3 -like enzymatic activity. NA accelerated caspase-3-like activity. Results of 12-h cultures were shown compared to freshly isolated blood neutrophils. One representative experiment out of three is shown. (c) All indicated caspase inhibitors (all used at $50 \mu \mathrm{M}$ ) completely blocked NA-mediated neutrophil apoptosis. One representative experiment out of three is shown (24-h cultures)

caspase-8 inhibitor Z-IETD-fmk and the caspase-9 inhibitor z-LEHD-fmk demonstrated similar blocking efficacies, consistent with previously published work suggesting that these caspases also play a role in neutrophil apoptosis. ${ }^{15,17}$ Taken together, NA accelerated spontaneous neutrophil apoptosis in a caspase-dependent manner in vitro.

NA-mediated reduction of cAMP levels associated with reduced phosphorylation of Bad at Ser 136. In initial experiments, we investigated whether pharmacological inhibition of calcineurin would block NA-mediated neutrophil death. Both FK506 (1-1000 ng/ml) and cyclosporin A $(1-1000 \mathrm{ng} / \mathrm{ml})$ had no effect on neutrophil viability in the presence and absence of NA (data not shown), excluding the possibility that calcium-mediated calcineurin activation is responsible for the pro-apoptotic effect of NA on neutrophils.

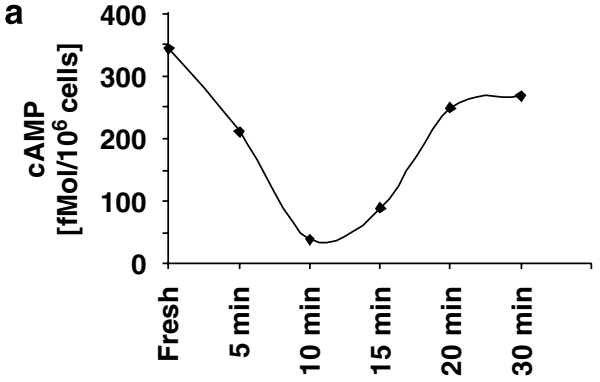

b

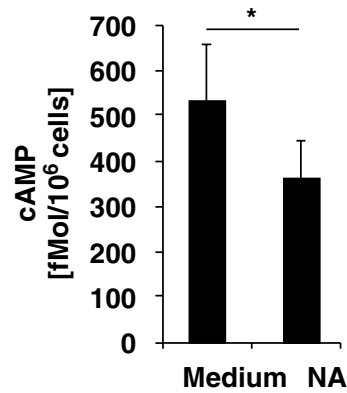

C

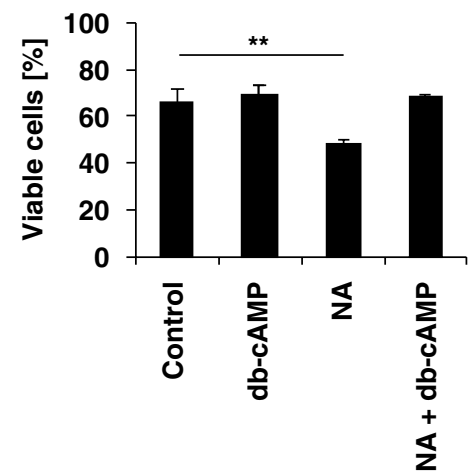

Figure 5 NA decreases intracellular cAMP levels in mature blood neutrophils. (a) NA stimulation of blood neutrophils resulted in a rapid reduction of intracellular cAMP levels. Maximal reduction was seen approximately 10 min upon NA stimulation. Concentration of CAMP increased thereafter, but did not reach the original levels. One representative experiment out of five is shown. (b) Reduced cAMP levels were also detected in neutrophils cultured for $6 \mathrm{~h}$ upon NA stimulation $(n=5)$. Data are expressed as means \pm S.D. ${ }^{*} P<0.05$. (c) The NA-mediated cytotoxic effect on neutrophils was reversible by increasing the levels of CAMP (exogenous db-cAMP was added at $100 \mu \mathrm{M}$ ). ${ }^{* *} P<0.01$

Besides intracellular calcium mobilization, GPR109A receptors have also been reported to inhibit adenylate cyclase activity. ${ }^{18}$ Therefore, we analyzed intracellular cyclic adenosine monophosphate (cAMP) levels in NAstimulated mature neutrophils. NA dramatically reduced cAMP levels within 10 min (Figure $5 \mathrm{a}$ ) and remained at this reduced level at least for $6 \mathrm{~h}$ (Figure $5 \mathrm{~b}$ ).

Previous reports suggested that cAMP levels regulate neutrophil apoptosis. ${ }^{19-21}$ We therefore asked the question whether the cell-permeable analogue of cAMP, dibutyryl cyclic adenosine monophosphate (db-cAMP), can reverse the pro-apoptotic effect of NA. Although db-cAMP had no significant effect on spontaneous neutrophil death, it completely abolished the NA-mediated cytotoxic effect (Figure $5 \mathrm{c}$ ). This suggested that the NA-mediated reduction 

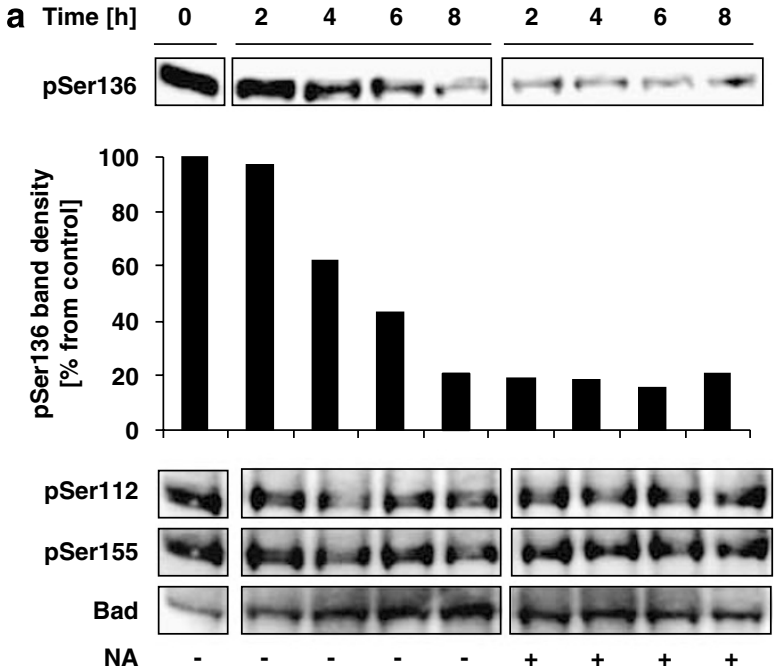

b

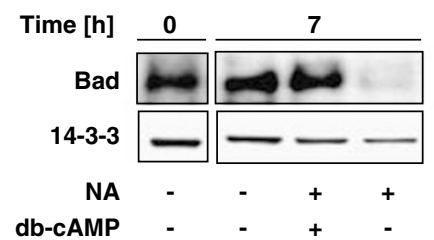

Figure 6 NA reduces phosphorylation of Bad at Ser 136 in mature blood neutrophils. (a) Neutrophils were cultured in the presence and absence of NA for the indicated time periods. Phospho Ser 112, Ser 136 and Ser 155 Bad, and total Bad were analyzed by immunoblotting. NA reduced Bad phosphorylation at Ser 136 that was quantified by densitometry. (b) 14-3-3 proteins in neutrophils were immunoprecipitated. Bad and 14-3-3 proteins were subsequently detected by immunoblotting. NA reduced the association between Bad and 14-3-3. This effect was not seen in the presence of exogenous db-cAMP. In both panels, representative immunoblots of at least two independent experiments are shown

in cAMP levels is a critical event for the subsequent induction of apoptosis.

Most effects of CAMP are mediated through activation of cAMP-dependent protein kinase $A(P K A)$. One potential target of PKA is Bad. $^{22,23} \mathrm{We}$, therefore, analyzed the phosphorylation pattern of Bad in neutrophils in response to NA stimulation. We observed that treatment of mature neutrophils with NA resulted in dephosphorylation of Bad at Ser 136, but not at Ser 112 or Ser 155, within $2 \mathrm{~h}$ (Figure 6a). Densitometry analysis indicated that 2-h NA treatment reduced Bad phosphorylation at Ser 136 approximately fivefold. These data suggested that Ser 136 phosphorylation is under the control of PKA in cultured neutrophils.

To demonstrate that attenuated Bad phosphorylation levels in NA-treated cells are functionally relevant, we investigated the physical association between Bad and 14-3-3 proteins. ${ }^{24}$ Neutrophil lysates were immunoprecipitated with anti-14-3-3 and blotted with anti-Bad and anti-14-3-3 antibodies. In freshly isolated as well as cultured neutrophils, Bad associated with 14-3-3 proteins, suggesting that the pro-apoptotic function of Bad was largely blocked. ${ }^{25}$ However, the interaction of Bad and 14-3-3 proteins decreased $7 \mathrm{~h}$ upon NA stimulation (Figure 6b). Importantly, db-cAMP completely prevented the NA-mediated disassociation of Bad and 14-3-3 proteins, in correlation with the prevention of NA-induced cell death (Figure 5c). Since the pharmacological inhibition of calcineurin

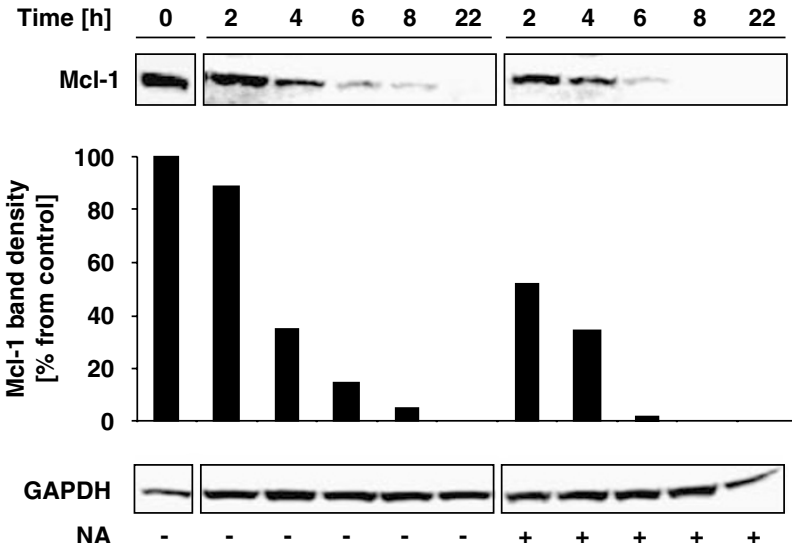

Figure $7 \mathrm{NA}$ accelerates $\mathrm{Mcl}-1$ degradation in mature blood neutrophils. Neutrophils were cultured in the presence and absence of NA for the indicated time periods. Mcl-1 expression was analyzed by immunoblotting. Mcl-1 declined over time, but more rapidly in the NA-stimulated neutrophils. Mcl-1 levels were quantified by densitometry. Representative immunoblots of three independent experiments are shown

had no effect on NA-mediated neutrophil death, these data suggested that NA-mediated reduced cAMP levels cause Bad dephosphorylation at Ser 136, resulting in its redistribution to mitochondria and consequently in increased apoptosis.

$\mathrm{Mcl}-1$ is rapidly degraded during neutrophil apoptosis and cAMP was shown to stabilize Mcl-1 protein. ${ }^{21} \mathrm{We}$, therefore, analyzed $\mathrm{Mcl}-1$ protein expression during spontaneous and NA-triggered neutrophil apoptosis. In NA-treated neutrophils, we observed markedly reduced $\mathrm{Mcl}-1$ levels following culturing cells for $2 \mathrm{~h}$ as well as at later time points (6 and $8 \mathrm{~h}$ ). In contrast, in non-treated neutrophils, $\mathrm{Mcl}-1$ levels declined less rapidly (Figure 7). Taken together, lowering cAMP levels by NA stimulation is associated with reduced Bad phosphorylation and accelerated $\mathrm{Mcl}-1$ degradation in mature neutrophils.

\section{Discussion}

The results of the current study suggest that mature neutrophils express functional GPR109A receptors, which have been known to inhibit lipolysis in adipocytes. ${ }^{1}$ Receptor expression in the neutrophilic lineage appears to be induced during the terminal differentiation phase, since a mixed population of immature neutrophils containing myeloblasts, promyelocytes, and metamyelocytes were GPR109A negative. Moreover, GPR109A expression was not seen in mature eosinophils, suggesting that it is not a general feature of all granulocytes. Recently, it has been demonstrated that the receptor is also expressed on macrophages and dendritic cells, but not on monocytes. ${ }^{4}$ Therefore, GPR109A receptors are expressed on some, but not all, leukocytes and may play a role in defense mechanisms against pathogens, at least within the innate immune system.

NA binds to both low- (GPR109B; HM74) and high-affinity (GPR109A; HM74A) receptors. Activation of GPR109A receptors occurs in an NA concentration range between $10^{-7}$ and $10^{-4} \mathrm{M}$, whereas GPR109B receptors require $10^{-3} \mathrm{M}$ NA for activation. ${ }^{2}$ Therefore, mature neutrophils express GPR109A receptors, since significant signal transduction 
(changes in $\left[\mathrm{Ca}^{2+}\right]_{i}$ ) and apoptosis induction was seen at $10^{-5} \mathrm{M}$ NA. However, we cannot exclude the possibility that these cells additionally express GPR109B receptors, in particular, since the antibody used to measure protein expression may recognize both receptors. The two receptors are highly homologous, displaying $96 \%$ identity at the protein level. ${ }^{2}$

Neutrophil apoptosis has previously been reported to be regulated by cAMP. For instance, agents and inflammatory stimuli that increase cAMP levels delayed spontaneous neutrophil apoptosis. ${ }^{19}$ The delay of apoptosis is also observed when the cell-permeable analogue of cAMP, dbcAMP, is added to neutrophils, directly demonstrating that CAMP mediates an antiapoptotic effect in these cells. ${ }^{20,21}$ Interestingly, elevation of cAMP promotes apoptosis in $\mathrm{T}$ cells, ${ }^{26,27}$ suggesting cell-type-specific differences. In agreement to previously published work performed in cell lines, ${ }^{28,29}$ we also observed reduced cAMP levels upon NA-mediated triggering of GPR109A receptors in mature neutrophils. This suggested a potential link to the NA-mediated pro-apoptotic effect observed in these cells. The fact that increasing the levels of cAMP by adding db-cAMP completely blocked the pro-apoptotic effect of NA supports this assumption.

PKA is a major target of CAMP $^{30}$ and may therefore be involved in cAMP-mediated antiapoptosis in neutrophils. However, the targets of PKA responsible for this effect remain to be determined. PKA is known to phosphorylate multiple targets, including Bad, a known pro-apoptotic member of the Bcl-2 family. ${ }^{22,23}$ Phosphorylated Bad is considered as being antiapoptotic, since it is bound in the cytosol to 14-3-3 proteins. ${ }^{25} \mathrm{We}$, therefore, hypothesized that NA-mediated decreased cAMP levels and subsequent decreased PKA activity may reduce $B$ ad phosphorylation levels. Indeed, in the presence of NA, Bad demonstrated decreased phosphorylation at Ser 136. Moreover, we obtained evidence that less Bad is sequestered in the cytosol by 14-3-3 proteins as the consequence of NA treatment. Interestingly, among the different phosphorylation sites of Bad, Ser 136 appears dominant in 14-3-3 binding. ${ }^{31,32}$ Taken together, our data suggest that less phosphorylated Bad as a consequence of NA stimulation is indeed pro-apoptotic and largely contributes to accelerated neutrophil apoptosis.

It has also been suggested that PKA may phosphorylate specific residues of the antiapoptotic $\mathrm{Bcl}-2$ family member $\mathrm{Mcl}-1$, leading to its stabilization. ${ }^{21} \mathrm{Mcl}-1$ plays an important role in neutrophil apoptosis. ${ }^{5}$ The more rapid degradation of $\mathrm{Mcl}-1$ in neutrophils in the presence of NA compared with nontreated cells could therefore be explained by reduced $\mathrm{Mcl}-1$ phosphorylation, leading to more rapid degradation. However, we cannot exclude the possibility that Mcl-1 degradation just correlated with the rate of apoptosis. Although the exact mechanisms of how NA induces neutrophil apoptosis remain to be determined, our initial data suggest that signal transduction events are involved in this pathway.

NA has been used as a lipid-lowering drug for decades. ${ }^{33}$ The primary action of NA is the inhibition of lipolysis in adipocytes, leading to an increase in HDL and a decrease in VLDL and LDL levels in blood. ${ }^{33}$ However, obesity is also considered as being a chronic inflammatory disorder. ${ }^{34}$ Limiting neutrophil numbers in chronic inflammatory re- sponses is an important anti-inflammatory mechanism. ${ }^{5}$ Therefore, the pro-apoptotic effect of NA on neutrophils may contribute to its overall beneficial effect in obese patients and GPR109A receptors continue to be promising targets for future pharmacotherapy.

\section{Materials and Methods}

Reagents. Anti-GPR109A (HM74a) rat mAb was from R\&D Systems Europe Ltd. (Abingdon, UK). NA, FK-506, and cyclosporin A were obtained from SigmaAldrich (Buchs, Switzerland). Fluorescein isothiocyanate (FITC)-conjugated NA was synthesized by Molecular Probes Europe BV (Leiden, The Netherlands). Anti-CD7 and anti-CD36 mAbs for immature neutrophil isolations, anti-Mcl-1 mAb, the Annexin $\mathrm{V}$ apoptosis detection kit and all caspase inhibitors (z-VAD-fmk, z-DEVDfmk, z-IETD-fmk, z-LEHD-fmk) were from BD Biosciences (Basel, Switzerland). Anti-CD16 mAb microbeads for eosinophil isolations were from Miltenyi Biotec $\mathrm{GmbH}$ (Bergisch Gladbach, Germany). Polyclonal anti-caspase-3 and all anti-Bad Abs were from Cell Signaling Technology Inc. (BioConcept, Allschwil, Switzerland). Polyclonal anti-14-3-3 $\beta$ (K-19) Ab was from Santa Cruz Biotechnology Inc. (Heidelberg, Germany). Anti-glyceraldehyde-3-phosphate dehydrogenase (GAPDH) $\mathrm{mAb}$ was from Chemicon International Inc. (Juro, Lucerne, Switzerland). Horseradish peroxidase (HRP)-conjugated secondary Abs were from Amersham Bioscience Europe GmbH (Freiburg, Germany). Phycoerythrin (PE) and tetramethylrhodamine isothiocyanate (TRITC)-conjugated donkey anti-rat secondary Abs were purchased from Jackson ImmunoResearch Laboratories (Milan Analytica, La Roche, Switzerland). Anti-Fas mAb (clone $\mathrm{CH}-11$ ) was obtained from MBL International Corporation (LabForce AG, Nunningen, Switzerland). PTX and fura-2-acetoxymethylester (fura-2/AM) were from Alexis (Lausen, Switzerland). Human GM-CSF was purchased from Novartis Pharma GmbH (Nürnberg, Germany). db-cAMP was provided by Biomol Research Laboratories Inc. (Plymouth Meeting, PA, USA). Fluo-3/AM was from Molecular Probes (Juro, Lucerne, Switzerland). UTP was from Boehringer Mannheim (Mannheim, Germany). All other chemicals were, unless stated otherwise, from Sigma-Aldrich.

Cells. Mature blood neutrophils were isolated from peripheral blood of healthy donors by Ficoll-Hypaque centrifugation. ${ }^{11,15,35,36}$ Briefly, peripheral blood mononuclear cells were separated by centrifugation on Ficoll-Hypaque (Seromed-Fakola AG, Basel, Switzerland). The lower phase, mainly granulocytes and erythrocytes, was treated with erythrocyte lysis solution $\left(155 \mathrm{mmol} / \mathrm{I} \mathrm{NH} \mathrm{N}_{4} \mathrm{Cl}\right.$, $10 \mathrm{mmol} / / \mathrm{KHCO}_{3}$, and $0.1 \mathrm{mmol} / / \mathrm{EDTA}, \mathrm{pH} 7.3$ ). The resulting cell populations contained greater than $95 \%$ mature neutrophils as assessed by staining with DiffQuik (Medion $\mathrm{GmbH}$, Düdingen, Switzerland) and light microscopy analysis.

Immature neutrophils were isolated from bone marrow aspirates with normal cellular morphology and distribution as described previously. ${ }^{15,16}$ Briefly, following centrifugation on a two-step discontinuous percoll density gradient, cells were negatively isolated using anti-CD7 and anti-CD36 mAbs to eliminate contaminating lymphoid and erythroid precursors. The resulting cell population contained $>97 \%$ cells of the neutrophil lineage as determined by myeloperoxidase staining, flow cytometric analysis of lineage-associated surface proteins, ${ }^{15}$ as well as with DiffQuik and light microscopy. The proportion of mature neutrophils was less than $5 \%$.

Peripheral blood eosinophils from control individuals were purified as described previously. ${ }^{37,38}$ The resulting cell populations contained more than $99 \%$ eosinophils as controlled by staining with Diff-Quik and light microscopy.

All individuals who donated blood or bone marrow aspirates signed a written informed consent agreement in accordance with the Declaration of Helsinki. The study was approved by the local medical ethics committee (the Kantonale Ethikkommission Bern).

Cell cultures. Neutrophils were cultured at $1 \times 10^{6} / \mathrm{ml}$ in RPMI-1640 containing $1 \%$ fetal calf serum (FCS) and $200 \mathrm{IU} / \mathrm{ml}$ penicillin $/ 100 \mu \mathrm{g} / \mathrm{ml}$ streptomycin (all from Life Technologies, Basel, Switzerland) in the absence or presence of the indicated concentrations of NA, FK-506, cyclosporine A, PTX, GM-CSF, caspase inhibitors, and db-cAMP for the indicated time periods. If not indicated, NA was used at $100 \mu \mathrm{M}$. HL-60 cells were cultured in Iscove's medium containing 10\% FCS.

\section{Cell-surface expression analysis}

Method 1. Neutrophils were stained with $2.5 \mu \mathrm{g} / \mathrm{ml}$ antihuman GPR109A mAb or isotype-matched control mAb. Binding of the primary Abs was visualized with 
PE-conjugated donkey anti-rat secondary $\mathrm{Ab}(1 / 100)$ using flow cytometric analysis (FACS-Calibur, BD Biosciences).

Methods 2 and 3. Cytospins of fresh or cultured neutrophils were made on noncoated slides. Cells were fixed in $4 \%$ paraformaldehyde at room temperature for 10 min and washed three times in PBS, pH 7.4. To prevent non-specific binding slides were incubated in blocking buffer (25\% human immunoglobulins, $25 \%$ normal goat serum, 25\% normal donkey serum, and 25\% BSA) at room temperature for $1 \mathrm{~h}$. Indirect immunostaining of GPR109A was performed at room temperature for $1 \mathrm{~h}$ with $2.5 \mu \mathrm{g} / \mathrm{ml}$ anti-GRP109A or isotype-matched control mAb. Cells were then incubated with TRITC-conjugated donkey anti-rat secondary $\mathrm{Ab}(1 / 100)$ in the dark at room temperature for $1 \mathrm{~h}$. In other experiments, cells were incubated with FITCconjugated NA for $30 \mathrm{~min}$. Specificity was controlled by pre-incubation of the cells with unlabeled $1 \mathrm{mM} \mathrm{NA}$ for $1 \mathrm{~min}$. In the latter experiments, nuclei were counterstained with propidium iodide. ${ }^{39}$ Slides were covered by coverslips and analyzed by confocal laser scanning microscopy (LSM 510, Carl Zeiss, Heidelberg, Germany) equipped with $\mathrm{Ar}$ and $\mathrm{HeNe}$ lasers.

\section{Intracellular calcium measurements}

Method 1. Intracellular ionized calcium concentrations $\left(\left[\mathrm{Ca}^{2+}\right]_{\mathrm{i}}\right)$ were assayed with a bulk spectrofluorometric assay using a Perkin Elmer LS50 spectrofluorimeter as described previously. ${ }^{40,41}$ In the PTX experiments, cells were pre-incubated with the inhibitor at the indicated concentrations and for the indicated times.

Method 2. $\left[\mathrm{Ca}^{2+}\right]_{\text {i }}$ were also assayed in single cells. Cells $\left(3 \times 10^{5}\right)$ were immobilized on glass cover slides for live-cell microscopy ${ }^{14}$ and loaded with $10 \mu \mathrm{M}$ fluo-3/AM for $30 \mathrm{~min}$. The attached cells were carefully washed and $200 \mu \mathrm{l}$ PBS supplemented with glucose $(2 \mathrm{mM})$ was added. Calcium measurements were performed in a $37^{\circ} \mathrm{C}$-chamber using a confocal laser scanning microscopy (LSM 510). Fluo-3- $\mathrm{Ca}^{2+}$ was excited at $514 \mathrm{~nm}$ and fluorescence emission was detected using a 560-nm longpass filter. Images were collected with a $\times 40,1.3$ NA Zeiss oil immersion objective. Single plane eight-bit images of $256 \times 256$ pixels representing $325.7 \mu \mathrm{m}$ in each dimension were recorded at intervals of $1.5 \mathrm{~s}$. After starting the recording process, agonists were applied at the indicated concentrations. From the resulting image series, signal intensities were calculated using the LSM510's built-in quantification software (Zeiss 510, version 2.5)

cAMP measurements. To rise cAMP to detectable levels, the phosphodiesterase inhibitor 3-isobutyl-1-methylxanthine $(100 \mu \mathrm{M})$ was added for $10 \mathrm{~min}$. For the time-dependent experiments, mature neutrophils $\left(1 \times 10^{6} / \mathrm{ml}\right)$ were placed into 48-well plates and cultured in the presence and absence of $100 \mu \mathrm{M} \mathrm{NA}$ for the indicated times. Intracellular levels of CAMP were analyzed using a commercial kit (Amersham) according to the recommendations of the manufacturer.

Determination of cell death and apoptosis. Cell death was assessed by uptake of $1 \mu \mathrm{M}$ ethidium bromide and flow cytometric analysis (FACSCalibur). ${ }^{11,15,35,36}$ To determine whether cell death was apoptosis, DNA fragmentation and redistribution of PS were measured. .11,15,35,36 $^{-1}$

Immunoprecipitation. Neutrophils $\left(5 \times 10^{6}\right)$ were lysed in a buffer containing $0.2 \%$ Nonidet P-40, and protein samples were immunoprecipitated with $2 \mu \mathrm{g}$ of anti-14-3-3 Ab followed by precipitation with A-Sepharose.

Immunoblotting. Gel electrophoresis and immunoblotting were performed as described previously. ${ }^{11,15,16,35,36}$ Briefly, after electrotransfer of the separated proteins, the filters were incubated overnight with anti-caspase-3 Ab, anti-Mcl-1 mAb, or anti-phospho-Bad Abs (all $1 / 1000$ ) at $4^{\circ} \mathrm{C}$ in TBS $/ 0.1 \%$ Tween $20 / 5 \%$ nonfat dry milk. For loading controls, stripped filters were incubated with anti-GAPDH mAb (1/2000) or anti-Bad Ab (1/1000). Filters were washed, incubated with the appropriate HRP-conjugated secondary Ab (Amersham), and developed by an ECL-technique (ECL-Kit, Amersham) according to the manufacturer's instructions.

Enzymatic caspase assay. Enzymatic caspase-3-like activity in cell-free extracts was measured as enzymatic conversion of the colorimetric substrate Ac-DEVD-pNA at $405 \mathrm{~nm}$ according to the manufacturer's instructions (QuantiZyme caspase-3 cellular activity assay kit, Biomol).

Statistical analysis. Statistical analysis was performed by using the MannWhitney U-test. If mean levels are presented, the standard deviation of the mean (S.D.) and the number $(n)$ of independent experiments are indicated in each case. A probability value of $<0.05$ was considered statistically significant.
Acknowledgements. This work was supported by the Swiss National Science Foundation (Grant no. 310000-107526), the Stanley Thomas Johnson Foundation, Bern, and OPO-Foundation, Zurich.

1. Tunaru S, Kero J, Schaub A, Wufka C, Blaukat A, Pfeffer $\mathrm{K}$ et al. PUMA-G and HM74 are receptors for nicotinic acid and mediate its anti-lipolytic effect. Nat Med 2003; 9: 352-355.

2. Wise A, Foord SM, Fraser NJ, Barnes AA, Elshourbagy N, Eilert M et al. Molecular identification of high and low affinity receptors for nicotinic acid. J Biol Chem 2003; 278: 9869-9874

3. Tavintharan S, Kashyap ML. The benefits of niacin in atherosclerosis. Curr Atheroscler Rep 2001; 3: 74-82.

4. Benyo Z, Gille A, Kero J, Csiky M, Suchankova MC, Nusing RM et al. GPR109A (PUMA-G/ HM74A) mediates nicotinic acid-induced flushing. J Clin Invest 2005; 115: 3634-3640.

5. Simon HU. Neutrophil apoptosis pathways and their modifications in inflammation. Immunol Rev 2003; 193: 101-110.

6. Colotta F, Re F, Polantarutti N, Sozzani S, Mantovani A. Modulation of granulocyte survival and programmed cell death by cytokines and bacterial products. Blood 1992; 80: 2012-2020.

7. Dibbert B, Weber M, Nikolaizik WH, Vogt P, Schöni MH, Blaser K et al. Cytokine-mediated Bax deficiency and consequent delayed neutrophil apoptosis: a general mechanism to accumulate effector cells in inflammation. Proc Natl Acad Sci USA 1999; 96: 1333013335.

8. Maianski NA, Mul FPJ, van Buul JD, Roos D, Kuijpers TW. Granulocyte colony-stimulating factor inhibits the mitochondria-dependent activation of caspase-3 in neutrophils. Blood 2002; 99: 672-679.

9. Brach MA, deVos S, Gruss HJ, Herrmann F. Prolongation of survival of human polymorphonuclear neutrophils by granulocyte-macrophage colony-stimulating factor is caused by inhibition of programmed cell death. Blood 1992; 80: 2920-2924.

10. Iwai K, Miyawaki T, Takizawa T, Konno A, Ohta K, Yachie A et al. Differential expression of bcl-2 and susceptibility to anti-Fas-mediated cell death in peripheral blood lymphocytes, monocytes, and neutrophils. Blood 1994; 84: 1201-1208.

11. von Gunten S, Yousefi S, Seitz M, Jakob SM, Schaffner T, Seger R et al. Siglec-9 transduces apoptotic and non-apoptotic death signals into neutrophils depending on the pro-inflammatory cytokine environment. Blood 2005; 106: 1423-1431.

12. Lorenzen A, Stannek C, Lang H, Andrianov V, Kalvinsh I, Schwabe U. Characterization of a G protein-coupled receptor for nicotinic acid. Mol Pharmacol 2001; 59: 349-357.

13. Lemoli RM, Ferrari D, Fogli M, Rossi L, Pizzirani C, Forchap S et al. Extracellular nucleotides are potent stimulators of human hematopoietic stem cells in vitro and in vivo. Blood 2004; 104: 1662-1670.

14. Kotevic I, Kirschner KM, Porzig H, Baltensperger K. Constitutive interaction of the P2Y2 receptor with the hematopoietic cell-specific $\mathrm{G}$ protein $\mathrm{G} \alpha 16$ and evidence for receptor oligomers. Cell Signal 2005; 17: 869-880.

15. Altznauer F, Martinelli S, Yousefi S, Thurig C, Schmid I, Conway EM et al. Inflammationassociated cell cycle-independent block of apoptosis by survivin in terminally differentiated neutrophils. J Exp Med 2004; 199: 1343-1354.

16. Martinelli S, Kostylina G, Niggli V, Baumann C, Fey MF, Wendel HG et al. Targeting survivin via PI3K but not $\mathrm{c}$-akt/PKB by anticancer drugs in immature neutrophils. Oncogene 2006; 25: 6915-6923

17. Altznauer F, Conus S, Cavalli A, Folkers G, Simon HU. Calpain-1 regulates Bax and subsequent Smac-dependent caspase-3 activation in neutrophil apoptosis. J Biol Chem 2004; 279: 5947-5957.

18. Soga T, Kamohara M, Takasaki J, Matsumoto S, Saito T, Ohishi $T$ et al. Molecular identification of nicotinic receptor. Biochem Biophys Res Commun 2003; 303: 364-369.

19. Rossi AG, Cousin JM, Dransfield I, Lawson MF, Chilvers ER, Haslett C. Agents that elevate cAMP inhibit human neutrophil apoptosis. Biochem Biophys Res Commun 1995; 217 892-899.

20. Krakstad C, Christensen AE, Doskeland SO. cAMP protects neutrophils against TNF- $\alpha-$ induced apoptosis by activation of CAMP-dependent protein kinase, independently of exchange protein directly activated by CAMP (Epac). J Leukoc Biol 2004; 76: 641-647.

21. Kato $T$, Kutsuna $H$, Oshitani N, Kitagawa S. Cyclic AMP delays neutrophil apoptosis via stabilization of Mcl-1. FEBS Lett 2006; 580: 4582-4586.

22. Harada H, Becknell B, Wilm M, Mann M, Huang LJ, Taylor SS et al. Phosphorylation and inactivation of BAD by mitochondria-anchored protein kinase A. Mol Cell 1999; 3: 413-422.

23. Affaitati A, Cardone L, de Cristofaro T, Carlucci A, Ginsberg MD, Varrone S et al. Essential role of A-kinase anchor protein 121 for cAMP signaling to mitochondria. J Biol Chem 2003; 278: $4286-4294$

24. Melander F, Andersson T, Dib K. Engagement of $\beta 2$ integrins recruits 14-3-3 proteins to c-Cbl in human neutrophils. Biochem Biophys Res Commun 2004; 317: 1000-1005.

25. Raff M. Cell suicide for beginners. Nature 1998; 15: 119-122.

26. McConkey DJ, Orrenius S, Jondal M. Agents that elevate cAMP stimulate DNA fragmentation in thymocytes. J Immunol 1990; 145: 1227-1230.

27. Kiefer J, Okret S, Jondal M, McConkey DJ. Functional glucocorticoid receptor expression is required for CAMP-mediated apoptosis in a human leukemic T cell line. J Immunol 1995; 155: $4525-4528$ 
28. Zhang Y, Schmidt RJ, Foxworthy P, Emkey R, Oler JK, Large TH et al. Niacin mediates lipolysis in adipose tissue through its G-protein coupled receptor HM74A. Biochem Biophys Res Commun 2005; 334: 729-732.

29. Zhou L, Tang Y, Cryan EV, Demarest KT. Human epidermoid A431 cells express functional nicotinic acid receptor HM74a. Mol Cell Biochem 2007; 294: 243-248.

30. Zaccolo M, Cesetti T, Di Benedetto G, Mongillo M, Lissandron V, Terrin A et al. Imaging the cAMP-dependent signal transduction pathway. Biochem Soc Trans 2005; 33: 1323-1326.

31. Masters SC, Yang H, Datta SR, Greenberg ME, Fu H. 14-3-3 inhibits Bad-induced cell death through interaction with serine-136. Mol Pharmacol 2001; 60: 1325-1331.

32. Chiang CW, Kanies C, Kim KW, Fang WB, Parkhurst C, Xie M et al. Protein phosphatase $2 \mathrm{~A}$ dephosphorylation of phosphoserine 112 plays the gatekeeper role for BAD-mediated apoptosis. Mol Cell Biol 2003; 23: 6350-6362.

33. Offermanns S. The nicotinic acid receptor GPR109A (HM74A or PUMA-G) as a new therapeutic target. Trends Pharmacol Sci 2006; 27: 384-390.

34. Tilg H, Moschen AR. Adipocytokines: mediators linking adipose tissue, inflammation and immunity. Nat Rev Immunol 2006; 6: 772-783.

35. Baumann R, Casaulta C, Simon D, Conus S, Yousefi S, Simon HU. Macrophage migration inhibitory factor delays apoptosis in neutrophils by inhibiting the mitochondria-dependent death pathway. FASEB J 2003; 17: 2221-2230.
36. Bruno A, Conus S, Schmid I, Simon HU. Apoptotic pathways are inhibited by leptin receptor activation in neutrophils. J Immunol 2005; 174: 8090-8096.

37. Yousefi S, Green DR, Blaser K, Simon HU. Protein-tyrosine phosphorylation regulates apoptosis in human eosinophils and neutrophils. Proc Natl Acad Sci USA 1994; 91: 1086810872.

38. Conus S, Bruno A, Simon HU. Leptin is an eosinophil survival factor. J Allergy Clin Immunol 2005; 116: 1228-1234

39. Simon D, Lindberg RLP, Kozlowski E, Braathen LR, Simon HU. Epidermal caspase-3 cleavage associated with interferon- $\gamma$-expressing lymphocytes in acute atopic dermatitis lesions. Exp Dermatol 2006; 15: 441-446.

40. Simon HU, Tsao PW, Siminovitch KA, Mills GB, Blaser K. Functional platelet-activating factor receptors are expressed by monocytes and granulocytes but not by resting or activated $\mathrm{T}$ and $\mathrm{B}$ lymphocytes from normal individuals or patients with asthma. J Immunol 1994; 153: 364-377.

41. Thomet OAR, Wiesmann UN, Blaser K, Simon HU. Differential inhibition of inflammatory effector functions by petasin, isopetasin and neopetasin in human eosinophils. Clin Exp Allergy 2001; 31: 1310-1320. 